\title{
Evaluación de variedades de Cucumis sativus L. a campo abierto en Tuxpan, Veracruz
}

\author{
Evaluation of varieties of Cucumis sativus L. a open field in Tuxpan, Veracruz \\ Hernández Mar, Brenda Mariana ${ }^{1}$, Figueroa Hernández, Víctor Manuel ${ }^{1}$, Castelán Ovando, Jorge \\ Alberto $^{1}$ \\ ${ }^{1}$ Facultad de Ciencias Biológicas y Agropecuarias de la Universidad Veracruzana. \\ Campus Tuxpan. \\ ${ }^{\circledR}$ Autor para correspondencia: brehernandez@uv.mx
}

Recibido: $21 / 08 / 2016$

Aceptado: 11/11/2016

\section{RESUMEN}

El pepino (Cucumis Sativus L.) es una hortaliza de alto potencial económico. En México se tiene registrado áreas de cultivo de esta especie en prácticamente todo el territorio nacional. Dada la importancia en la actualidad, se realizan cada vez más estudios referentes a la evaluación de las variedades para hacer constar su adaptabilidad a climas y comprobar rendimientos, tolerancias a plagas y enfermedades. El objetivo del experimento fué evaluar diferentes variedades de pepino al norte del estado de Veracruz. El estudio se realizó en la Facultad de Ciencias Biológicas y Agropecuarias de la Universidad Veracruzana, Campus Poza Rica-Tuxpan. Los tratamientos utilizados fueron Esparón, Macario, 22-960, Alcázar, Primavera y Paraíso. El diseño utilizado fue bloques al azar con 4 repeticiones. Utilizando como variables de respuesta diámetro del tallo, longitud de la planta, hojas, floración, producción y rendimiento. Con un diámetro de $1.08 \mathrm{~cm}$ y una longitud de $381.6 \mathrm{~cm}$ destaca el tratamiento 22-960. Con una media en la floración de 23.5, el tratamiento Esparón fue el mejor. Para la media de hojas con 36.0, el tratamiento 22-960. Y liderando con una producción de 17.31 toneladas y un rendimiento de 141.31 cajas de producto súper selecto, el tratamiento 22-960. Por lo anterior concluimos que el tratamiento 22-960 obtuvo una mayor adaptabilidad para la zona al norte del estado de Veracruz.

Palabras clave: Pepino, variedades, cultivo. 


\begin{abstract}
Cucumber (Cucumis sativus L.) is a vegetable of high economic potential. In Mexico it has registered growing areas this species in almost all the national territory. Given the importance today, more and more studies on the evaluation of the varieties are made to register their adaptability to climates and check yields, tolerance to pests and diseases. The aim of the experiment was to evaluate different varieties of cucumber north of Veracruz state. The study was conducted at the School of Biological and Agricultural Sciences of the Universidad Veracruzana Poza Rica-Tuxpan Campus. The treatments were Esparon, Macario, 22-960, Alcazar, Spring and Paradise. The design was randomized block with 4 replications. As response variables using stem diameter, length of the plant, leaves, bloom, production and performance. With a diameter of $1.08 \mathrm{~cm}$ and a length of $381.6 \mathrm{~cm}$ stands 22-960 treatment. With an average of 23.5 in bloom, the Esparon treatment was the best. For leaves with 36.0 average, 22-960 treatment. And leading with a production of 17.31 tons and a yield of 141.31 product boxes super select, treatment 22-960. Therefore we conclude that treatment 22-960 obtained greater adaptability to the area north of Veracruz state.
\end{abstract}

Keywords: Cucumber, variety, cultivation.

\section{INTRODUCCIÓN}

Rivera, (2006) menciona que el progreso que ha experimentado la producción de hortalizas en los últimos años ha sido el uso de nuevas variedades con cualidades diferentes que han venido desarrollando las compañías semilleras, como resultado de nuevas técnicas, exigencias agrícolas e industriales y de problemas derivados de la presencia de nuevas plagas y enfermedades, esto ha permitido incrementar los rendimientos y además presentan plantas más vigorosas, mayor cuajo de frutos, mayor producción, más resistencia a plagas y enfermedades, precocidad, uniformidad en la maduración, más consistencia, mejor color y homogeneidad en el calibre de los frutos. Jaramillo et al., (2013) manifiesta que el productor con el fin de garantizar el éxito en el cultivo debe comprar semillas certificadas, que sean producidas por compañías acreditadas y que estén apropiadamente empacadas; además, para asegurar la calidad de lo que está comprando, la etiqueta debe incluir las características del material y las condiciones de almacenamiento de la semilla, incluyendo una evaluación que describa su rendimiento y productividad en las condiciones agroecológicas donde se va a sembrar. La elección de un hibrido o variedad depende de las necesidades del productor, del comercializador y del consumidor. El productor selecciona un material de alto rendimiento, de buena apariencia, buen precio, adaptado a sus condiciones agroecológicas, con tolerancia a enfermedades, lo que asegura -si se da un manejo agronómico adecuado- altas producciones y menor uso de pesticidas para el manejo de plagas y enfermedades, con una vida poscosecha adecuada para resistir la manipulación y soportar el transporte a los centros de comercialización. Escalonada, et al, (2009) aduce que en la 
actualidad existe un permanente remplazo de buenas variedades por otras con mejores atributos, y el ritmo de cambio es tan dinámico que se hace difícil componer una lista de las variedades en uso, de ahí la importancia de consultar a los técnicos que conocen el área sobre cual variedad usar para un objetivo específico, en un lugar determinado. Los consumidores de frutos de pepino consideran aspectos importantes como son la forma, color, tamaño y textura, sin embargo en regiones de poco desarrollo estas características son menos exigentes, tanto que no limitan la comercialización. Según Clemente, (2012) en el ámbito hortofrutícola el concepto de calidad, en general, es muy importante, amplio y complejo y subjetivo desde un punto de vista práctico o comercial podemos entender a la calidad como "un conjunto de propiedades y características de un producto, bien $\mathrm{o}$ servicio que le confiere la aptitud para satisfacer necesidades declaradas de los usuarios, puede considerar características externas como la forma, color y tamaño. En base a lo anterior, se plantearon el Objetivo el Evaluar seis diferentes variedades de pepino bajo manejo a campo abierto, en la temporada agrícola 2014 - 2015, en Tuxpan, Veracruz.

\section{MATERIALES Y MÉTODOS}

El experimento se realizó en el área de producción de la Facultad de Ciencias Biológicas y Agropecuarias zona Poza Rica-Tuxpan durante la temporada agrícola 2014-2015, ubicada en las coordenadas $20^{\circ} 57^{\prime} 46^{\prime \prime} \mathrm{LN}$ $97^{\circ} 24^{\prime} 01^{\prime \prime}$ 'LW, el clima predominante es cálido-húmedo con lluvias en verano. La precipitación media anual es de 1,700 mm y se concentra en los meses de julio a octubre. La temperatura media anual oscila entre los $25^{\circ} \mathrm{C}$, con máximas de hasta $41{ }^{\circ} \mathrm{C}$ en el verano y mínimas de 9 ${ }^{\circ} \mathrm{C}$ en el invierno.

Diseño de bloques al azar, con 6 tratamientos y 4 repeticiones, la parcela experimental estuvo constituidas por 6 surcos de $12 \mathrm{~m}$ de largo, con separación entre ellos $1.5 \mathrm{~m}$. Las parcelas útiles las formaron los 4 surcos centrales, de los que se eliminaron $1 \mathrm{~m}$ en cada extremo y el mismo modo los dos surcos laterales con la finalidad de eliminar el efecto orilla. El área del experimento estuvo

provista de acolchado plástico blanco/negro y un sistema riego por goteo.

Se evaluaron 6 tratamientos los cuales consisten en diferentes híbridos de pepino Slicer; estos híbridos de diferentes casas comerciales. Como tratamientos que se consideraran para la evaluación de los híbridos serán los materiales siguientes: Esparón, Macario, 22-960, Alcázar, Primavera y Paraíso como testigo el cual es el hibrido que comercialmente se utiliza con mayor frecuencia en la zona. Las Variables de respuesta utilizadas fueron: Geminación y emergencia, Diámetro del tallo, Longitud de la planta, Precocidad en la floración, Rendimiento/há.

\section{RESULTADOS}

A los resultados obtenidos se les realizó el análisis de varianza y la prueba de comparación de medias, destacándo las variables siguientes: Número de floración, para esta variable existieron diferencias estadísticamente significativas, las variedades con más altos índices de floración fueron Esparón, Primavera y 22-960 con 23.5, 23.0 y 
21.25 respectivamente destacando entre ellas la variedad Esparón, ya que esta variedad presento mayor precocidad en la floración, haciéndolo a los 27 dds, lo que representa 3 días de diferencia antes que el resto de las variedades, esto se atribuye a que dentro de las características reportadas dentro la ficha técnica de la variedad es un material vegetal en el que se potencializa la floración cuando su época de siembra va de calor a frio, siendo este el caso. Las variedades Macario, Alcázar y Paraíso mostraron los índices más bajos de floración con 12.5, 7.75 y 7.5 respectivamente, lo que se aprecia en la Fig. 1.. Estos resultados son comparables con Perdomo, (2013) que trabajó con las variedades Tropi-cuke 1l, Llano verde, Tropicuke 1, conquistador y Diamante y reporta en su análisis para esta variable una precocidad de 23 días a floración mientras que las variedades más tardías presentaron un proceso de floración a los 25 días, representando 2 días de diferencia en la precocidad de la floración.

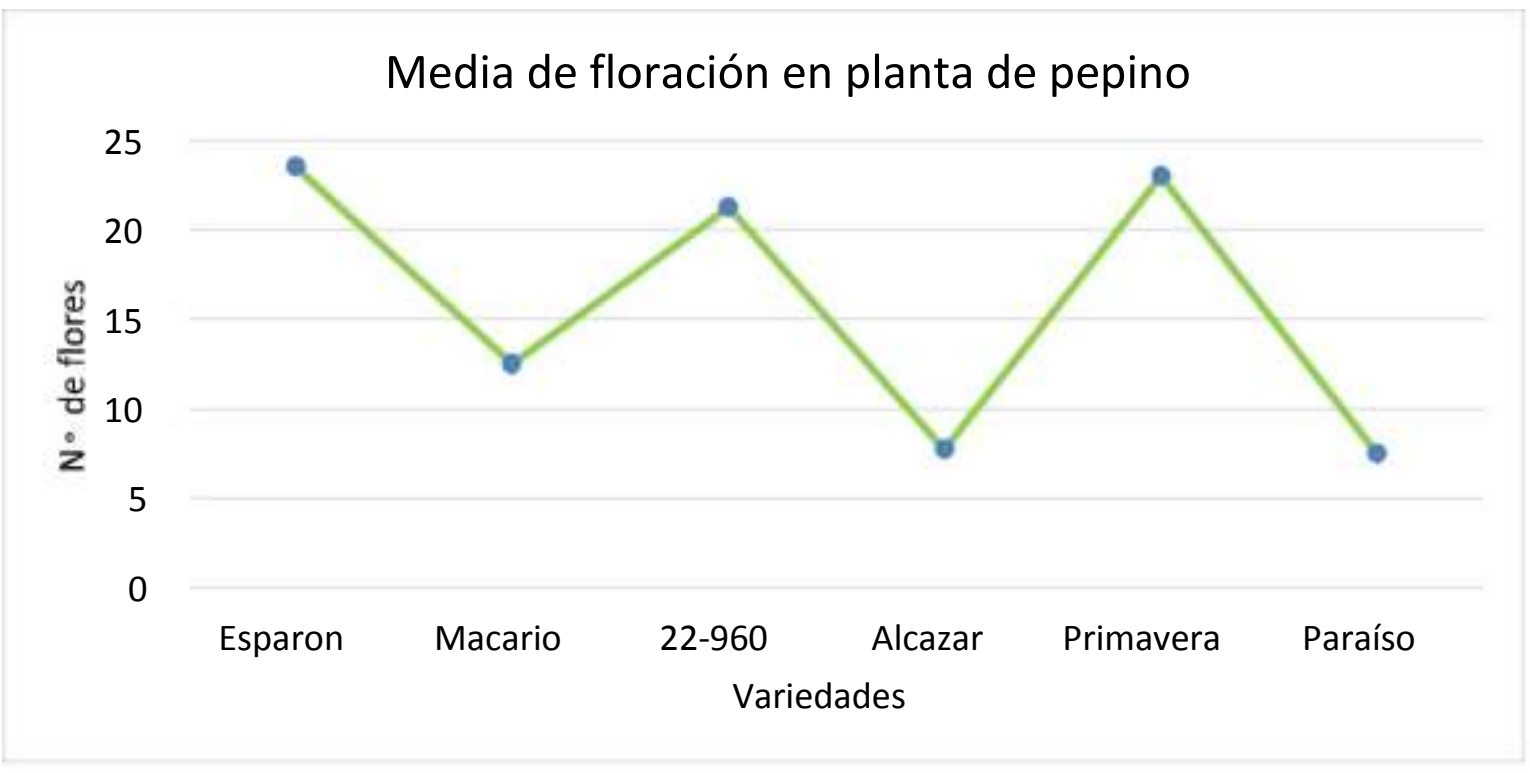

Figura 1. Prueba de comparación de medias de la variable de respuesta número de flores.

En la variable Producción, se observaron las siguientes diferencias significativas, el tratamiento 22-960 fue el más alto con un total de 17.31 ton/ha ya que fue la variedad que mejor se adaptó a la climatología de la zona, seguidos de los tratamientos Macario y Esparón con 15.29 y 14.98 ton/ha respectivamente. El tratamiento Primavera mostró un total de 10.53 ton/ha. Los tratamientos con la producción más baja fueron los tratamientos Paraíso y Alcázar con un total de 8.31 y 5.79 ton/ha respectivamente, siendo las variedades que tardaron más en adaptarse a la climatología de la zona. Estos datos del tratamiento 22-960 coinciden con SIAP, (2013) que nos indica que la media total de Veracruz oscila entre las 17.54 ton/ha.

Revista Científica Biológico Agropecuaria Tuxpan 4 (2) 
Para la Variable Rendimiento, se encontró que el tratamiento que

consistentemente mostró mejores tamaños de corte fue el tratamiento 22960; seguido del tratamiento Macario, tratamientos que mostraron los tamaños promedios de comercialización más constantes (Fig. 2).

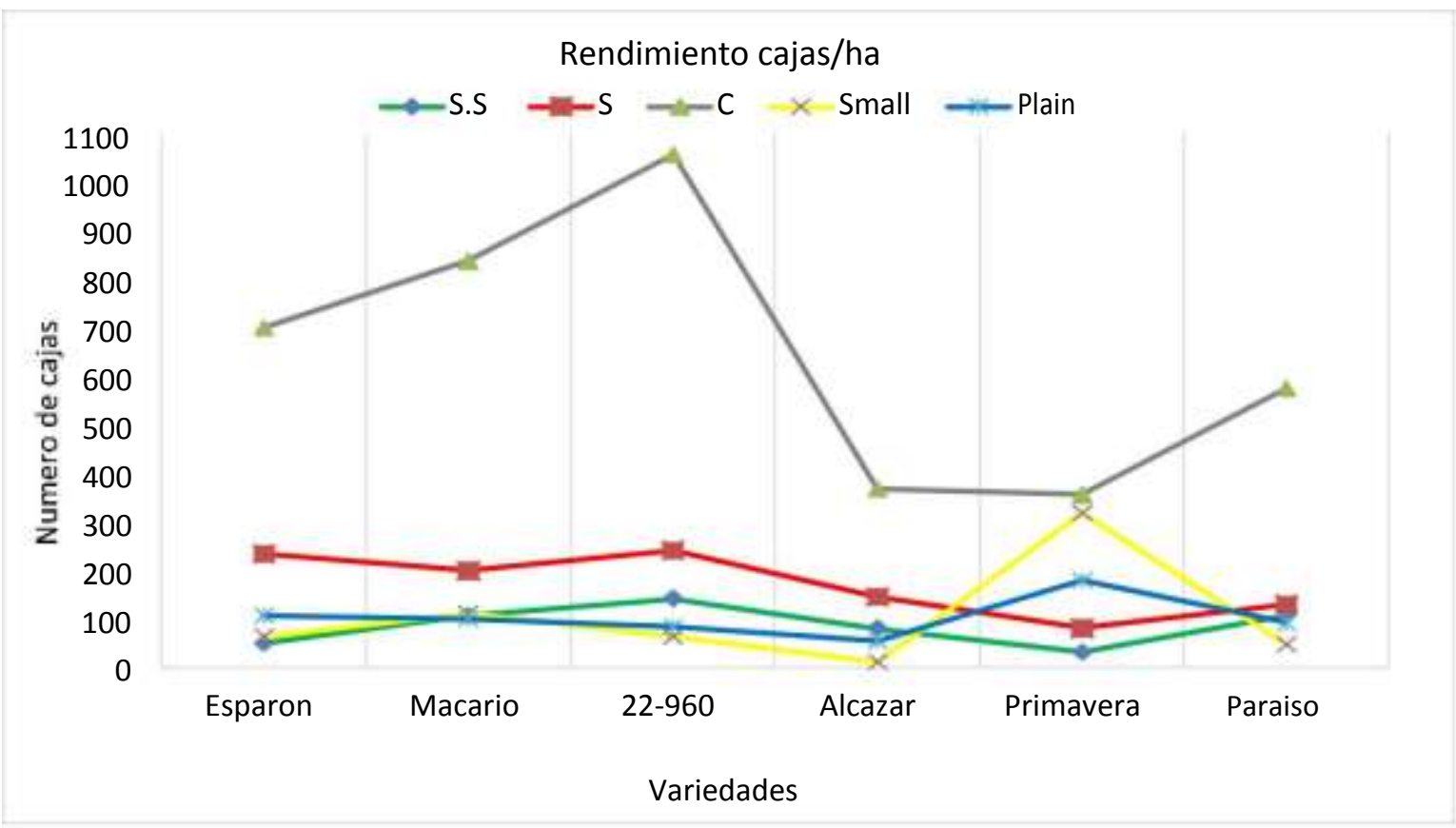

Figura 2. Rendimiento de las diferentes variedades.

\section{CONCLUSIONES}

En general, La variedad 22-960 presentó las mejores cualidades. Los aspectos como diámetro de tallo, la longitud de la planta, el número de hojas, porcentajes de germinación y emergencia son determinantes en la productividad de los cultivos en lo que para este caso la variedad 22-960 destacó entre las demás, seguida la variedad Macario, sin embargo, la variedad primavera presentó mayor robustez en sus tallos por lo que se considera como un material vegetal de excelente soporte.
Las variedades Esparón, Macario y 22-960 presentaron mayor precocidad en la floración, aspecto muy importante ya que permite aprovechar las ventanas de mercado y comercializar a precios más atractivos. Las variedades que presentaron mejor patrón de adaptación para las condiciones climáticas de Tuxpan, Veracruz fueron 22-960 y Esparón ya que de manera general son plantas que presentan una buena estructura, anatómicamente hablando y con una producción considerada dentro de la media nacional de 17 ton/ha. El rendimiento de la variedades 22-960 y Macario supera al resto, ya que en su 
mayoría presentan frutos de excelente y buena calidad considerados dentro de las tallas super select y select, clasificados como frutos con calidad de exportación.

\section{LITERATURA CITADA}

Alvarado, R. B.; Trumble J. T. 1990. El manejo integrado en las plagas de tomate en Sinaloa. Folleto técnico. Confederación de Asociaciones Agrícolas del estado de Sinaloa. University California. 16 pp.

Armenta, G. T. 1988. El cultivo de pepino (Cucumis sativus L.). Resumen de Guía para la producción de hortalizas. Cali (Colombia). 106-106 pp.

Caldari, P. J. 2007. Manejo de luz en invernaderos. Los beneficios de luz de calidad en cultivo de Hortalizas. Simposio internacional de invernaderos, México. 4pp.

Cabrera, A. Arzuaga J.; Mojena M. 2007. Desbalance nutricional del suelo y efecto sobre el rendimiento de tomate (Solanum lycopersicum L.) y pepino (Cucumis sativus L.) en condiciones de cultivo protegido. Cultivos Tropicales, 28(3):9197 pp. Contreras G. L.; Macías D. R., Grijalva D. S. A.; Robles C. F. 2011. Evaluación del efecto de la fecha de siembra en la productividad y calidad de híbridos de pepino europeo bajo condiciones de invernadero en el noroeste de sonora. Biotecnia, 13 (1): 29-36 pp. https://doi .org/10.18633/btv13i 1.
Enza zaden. 2014. Catálogo de variedades del cultivo de pepino. Comercializadora internacional de semillas. Ficha técnica de variedades. Consultado el 25 de mayo de 2014. Disponible en: (http://www.enzazaden.com. $\mathrm{mx}$ )

Gálvez, H. 2004. El cultivo de pepino en invernadero. Manual de Producción Hortícola en Invernadero. $2^{\mathrm{a}}$ ed. Castellanos. Intagri. Celaya, México.282-293 pp.

García, N. 2012. Comparativo de productividad de tres variedades de pepino (Cucumis sativus L.) en el alto mayo. Tesis de licenciatura Universidad Nacional De San Martín-

Tarapoto Facultad De Ciencias Agrarias Departamento Académico Agrosilvo Pastoril Escuela Academico-profecional De Agronomía. Tarapoto, Perú. $46 \mathrm{pp}$.

López, E. J.; Rodríguez, J.; Huez, M.; Garza, S.; Jiménez, J.; Leyva, E. 2010. Producción y calidad de pepino (Cucumis sativus L.) bajo condiciones de invernadero usando dos sistemas de poda. Tesis de licenciatura. Universidad de Sonora, Departamento de Agricultura y Ganadería. Hermosillo, Sonora. México.

Perdomo, M. J. 2013 Evaluación del potencial de rendimiento $\mathrm{y}$ adaptabilidad de cinco de pepino (athivames sativus L.) en dos 
localidades de la región de

Guatemala. Zacapa,

Guatemala. 36 p.

Reche, M. J. 2011. Cultivo de pepino en invernadero. Ministerio de Medio Ambiente y Medio Rural y Marino. ISBN: 978-84-491-1112$9.50 \mathrm{pp}$.

Té, E. 2008. Producción orgánica de tres variedades de pepino bajo condiciones de invernadero. Tesis de licenciatura. Universidad Autónoma de Querétaro. Facultad de Ingeniería. México.

Vasco M. R. 2003. El cultivo de pepino bajo invernadero. In: Técnicas de producción en cultivos protegidos. F. F. Camacho (ed), Caja Rural Intermediterranea. Cajamar. Almeria, España. 691 - 722

pp. 
Copyright (c) 2016 Brenda Mariana Hernández Mar, Victor Maruel Figueroa Hernández,

Jorge Alberto Castelán Ovando

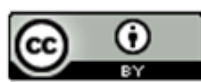

Este tex to está protegido por una licencia licencia Creative Commons $\underline{4.0}$

\begin{abstract}
Usted es libre para Compartir —copiar y redistribuir el material en cualquier medio o form ato-y Adaptar el documento -remezclar, transformar y crear a partir del material- para cualquier propósito, incluso para fines comerciales, siempre que cumpla la condición de:

Atribución: Usted debe dar crédito a la obra original de manera adecuada, proporcionar un enlace a la licencia, e indicar si se han realizado cambios. Puede hacerlo en cualquier form a razonable, pero no de form a tal que sugiera que tiene el apoyo del licenciante o 10 recibe por el uso que hace de la obra.

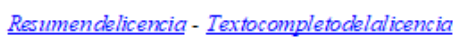

